\title{
Design and Development of a Path-Tracking System Based on Radio Frequency Identification Sensor for Educational Toy Robot (EDOT)
}

\author{
Martinus Bagus Wicaksono \\ Department of Mechatronic Product Design, \\ Politeknik Mekatronika Sanata Dharma, Yogyakarta, Indonesia \\ Corresponding Author: baguswicax@yahoo.co.id
}

(Received 31-01-2019; Revised 21-05-2019; Accepted 21-05-2019)

\begin{abstract}
This paper offers the design and development of a path-tracking system based on Radio Frequency Identification (RFID) sensors. This Path-tracking system will be used as a navigation system on EDOT. The EDOT requires a navigation system because it must be able to drive from the starting point to the predetermined end point automatically. This path-tracking system uses RFID sensors to detect RFID cards which have been arranged as a path. And then the EDOT will pass through the path consisting of some RFId cards. EDOT is a solution of a previous system, called Line-Follower, which uses infrared as a sensor to detect lines to guide a robot to go towards its destination point. The path-tracking system used by EDOT can work more efficiently in detecting the path to be traversed than other robots using the line follower system with infrared sensors or LDR (Light Dependent Resistor).
\end{abstract}

Keywords: path-tracking, RFID, EDOT, robot 


\section{International Journal of Applied Sciences and Smart Technologies}

Volume 1, Issue 2, pages 169-178

p-ISSN 2655-8564, e-ISSN 2685-9432

\section{Introduction}

In recent years many children's toys have used robotic technology [1]. These toy robots are used to develop the ability of children, especially toddlers, to think logically. One of the technologies used is the line-follower robot, where the robot can go along the line that has been prepared beforehand.

In the game, the line as a robot guide when driving has been prepared first. This line is prepared so that it can guide the robot from one point (start point) to the destination point (endpoint). In general, the sensor used to detect the presence of lines is an infrared sensor. In fact, the performance of this sensor in detecting lines is strongly influenced by two things, namely the distance of the sensor to the line and the presence of external light. As well as in the game there are shapes and length of the track that cannot be changed according to the player's wishes. Thus the game will be limited by a number of things above.

This paper offers a design of a path-tracking system based on RFID sensors to detect RFId cards that have been arranged to form a path that the robot will pass. This system is more effective in reading paths that will be traversed by robots when compared to the line follower system that uses infrared sensors. In line follower that uses a light sensor (LED and Photodiode); the sensitivity of the sensor will be greatly influenced by the light around the robot [2]. Another line follower system that uses the LDR sensor as a tracking and navigating sensor on the robot also has almost the same recommendation, which is greatly influenced by the distance of the sensor to the detected line and also the reflection of light from the surrounding environment [3]. In other applications, the RFId sensor is used to detect the identity of the car that will enter the parking area and provide information on the parking position of the car [4]. For the path-tracking system using the RIFd sensor, it will use a standard RFId sensor on the market. As well as the RFId card that will be used as a robot track compiler, it will use a standard RFId card on the market. This system will use the Arduino Nano as a control system. All needed components will use standard components on the market to reduce prices and accelerate the process of making a Path-tracking system. 


\section{International Journal of Applied Sciences and Smart Technologies}

Volume 1, Issue 2, pages 169-178

p-ISSN 2655-8564, e-ISSN 2685-9432

This paper is written in the following structure: in part 2 it will describe the design of the path-tracking system on EDOT. The method of making Path-tracking system will be described in section 3. While the results of the research and discussion will be written in section 4 .

\section{Design}

In this section, we explain about the design of our proposed system. RFId sensors are sensors that use radio wave-based technology. This sensor will detect objects called RFId cards. The communication method of these two objects is with the RFId sensor emitting waves that will trigger the power and clock on the RFId card to send data in the form of the identity that has been written on the RFId card.

In this design the RFId card will be used and arranged as a path, RFId sensors that will be used to detect trajectories, Arduino Nano as a control system, DC motor driver used as a motor movement regulator, the DC geared motor is used as the main movements generator of the robot. The block diagram representing the process is shown in the figure 1 below. This path-tracking system is designed using standard components on the market. The purpose is to simplify the replacement of components if damage occurs due to misuse. Another reason is that components on the market will be cheaper compared to customized components.

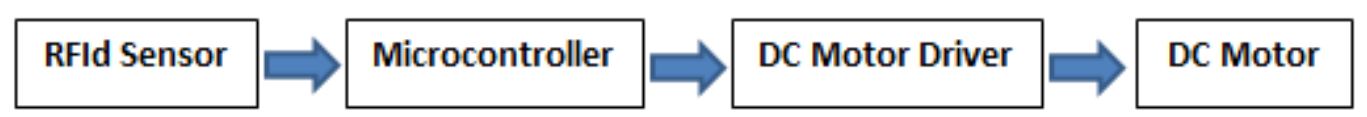

Figure 1. Block Diagram of the path tracking system

In some applications, RFId cards are used to store personal identity data. And in the existing system the card will be used for student attendance detector [5]. In this design, RFId path-tracking system, the path will use some RFId cards that have been given a different identity according to the required command, as shown in table 1.The cardsare RFId card $125 \mathrm{KHz}$ as shown in the figure 2 below. 


\section{International Journal of Applied Sciences and Smart Technologies}

Volume 1, Issue 2, pages 169-178

p-ISSN 2655-8564, e-ISSN 2685-9432

Table 1. List of RFId card identity

\begin{tabular}{ccc}
\hline No & Card identity & Command \\
\hline 1 & 0000001990 & Forward \\
2 & 0000002350 & Turn left \\
3 & 0000002357 & Turn right \\
4 & 0000002334 & Stop \\
\hline
\end{tabular}
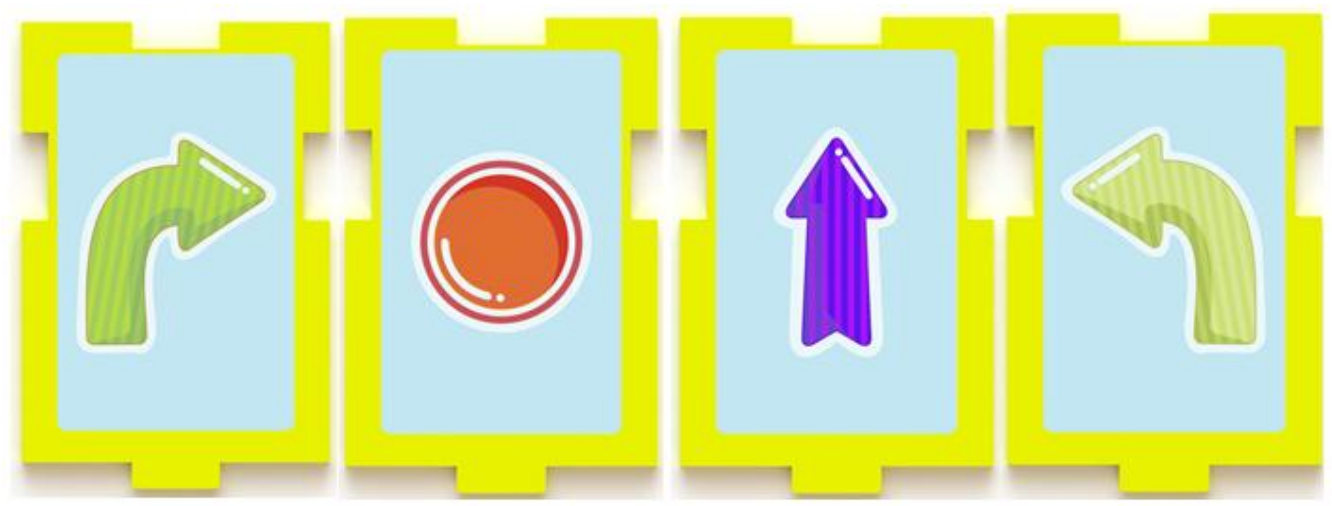

Figure 2. RFId card: turn right, Stop, Forward, Turn Left

The RFId Sensor RC522, as shown in Figure 3, is used to detect the RFId cards which have their own identity. The sensor is installed at the bottom of the robot, so that it can directly detect the RFId card which is arranged as the path that EDOT will pass. The position of the sensor is placed between the main wheels to make the EDOT move easier along the pre-arranged track.

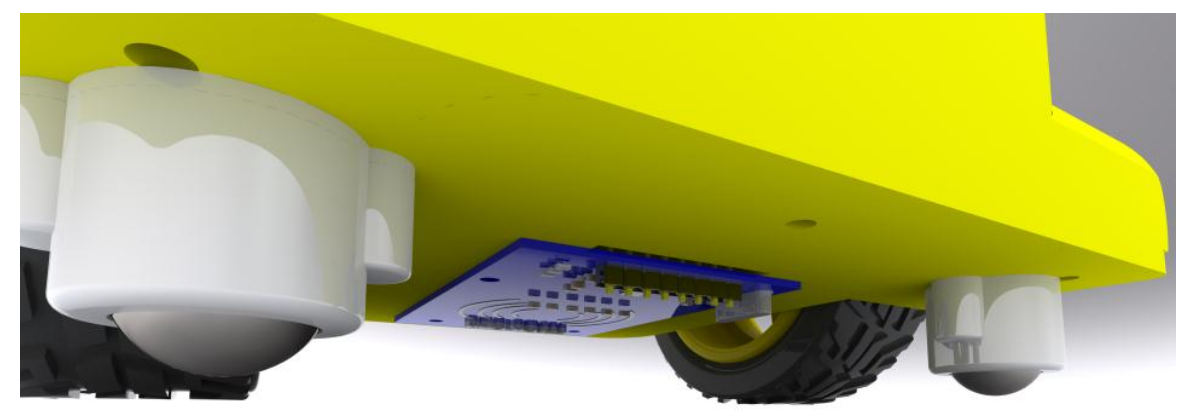

Figure 3. RFId Sensor

A microcontroller, Arduino Nano, is used to control EDOT based on reading data on an RFId card carried out by an RFId sensor. The program has been written on a 


\section{International Journal of Applied Sciences and Smart Technologies}

Volume 1, Issue 2, pages 169-178

p-ISSN 2655-8564, e-ISSN 2685-9432

microcontroller that will control the rate of EDOT following the prepared path. Data received by the RFId sensor which is the result of reading the RFId card will be an input to the microcontroller. The microcontroller will send the command to the DC motor driver which will adjust the rotation of the DC motor as the main driver of the EDOT as shown in Figure 4.
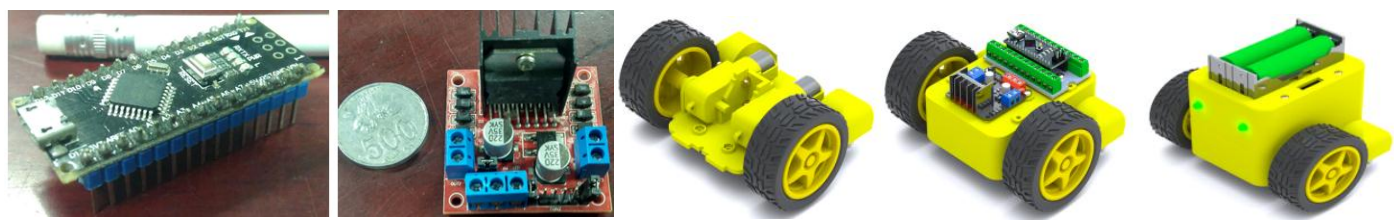

Figure 4. Arduino Nano, DC Motor Driver (L298N), part of EDOT

Figure 5 shows the overall shape of EDOT that uses the RFId sensor as a pathtracking system. As discussed in the previous paragraph, the RFId sensor is placed on the bottom surface of the EDOT facing the track. This is so that the sensor can detect the track properly. Figure 6 shows EDOT is moving following the prepared path. RFId cards must be arranged so that they give the same direction as the arrows on the RFId card.

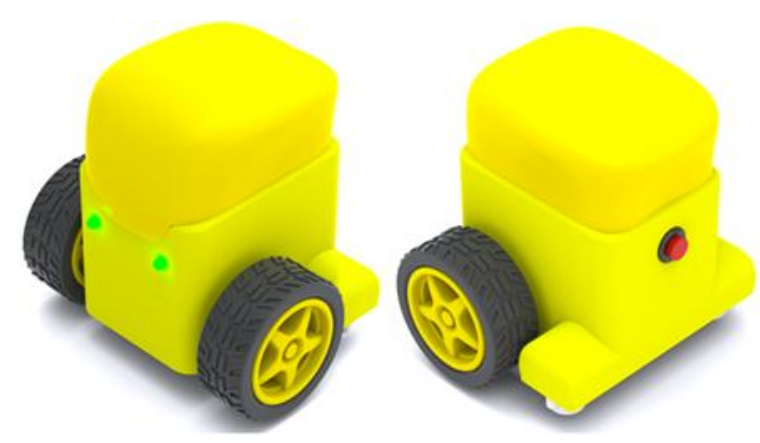

Figure 5. The EDOT 


\section{International Journal of Applied Sciences and Smart Technologies}

Volume 1, Issue 2, pages 169-178

p-ISSN 2655-8564, e-ISSN 2685-9432

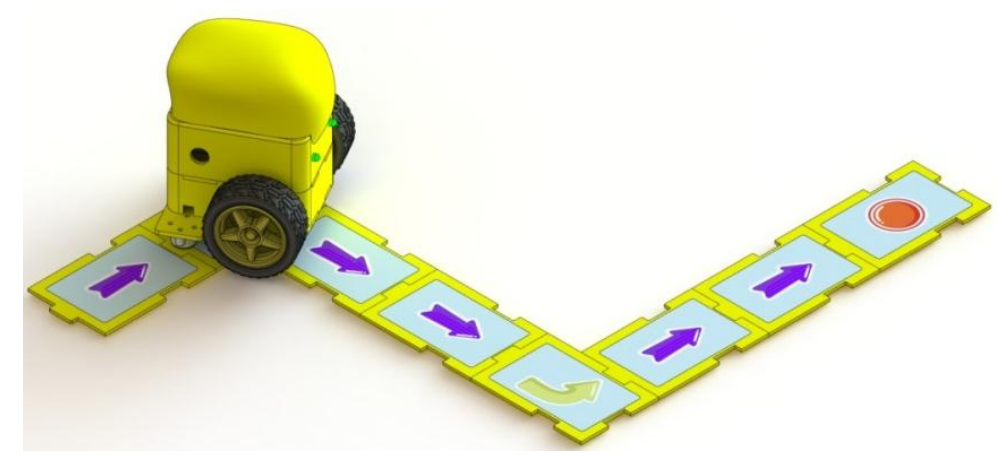

Figure 6. EDOT on the Path

\section{Method}

This section is devoted for the research method. In order to validate the system, some experiments in path-tracking have been conducted. The purpose of the experiments is to know the responses of the system in tracking a path. Some RFId cards with different identities were used to check the system accuracy in detecting the path. The procedure of the experiment is as follows:

1. Set the identity of some RFId cards based on the command (forward, turn left, turn right, stop),

2. Make 5 cards in every command,

3. Run the path-tracking system,

4. Detect the RFId card,

5. Vary the distance of the sensor to the card, and

6. Record the observations of the system.

By detecting the different card and varying the distance of the sensor to the card, the data in table 2 to table 7 could be obtained.

Table 2. Detection distance of $1 \mathrm{~mm}$

\begin{tabular}{lll}
\hline No & Type of command & $\begin{array}{l}\text { Detection } \\
\text { Result }\end{array}$ \\
\hline 1 & Forward & Success \\
2 & Turn Left & Success \\
3 & Turn Right & Success \\
4 & Stop & Success \\
\hline
\end{tabular}

Table 3. Detection distance of $10 \mathrm{~mm}$

\begin{tabular}{lll}
\hline No & Type of command & $\begin{array}{l}\text { Detection } \\
\text { Result }\end{array}$ \\
\hline 1 & Forward & Success \\
2 & Turn Left & Success \\
3 & Turn Right & Success \\
4 & Stop & Success \\
\hline
\end{tabular}




\section{International Journal of Applied Sciences and Smart Technologies}

Volume 1, Issue 2, pages 169-178

p-ISSN 2655-8564, e-ISSN 2685-9432

Table 4. Detection distance of $20 \mathrm{~mm}$

\begin{tabular}{lll}
\hline No & Type of command & $\begin{array}{l}\text { Detection } \\
\text { Result }\end{array}$ \\
\hline 1 & Forward & Success \\
2 & Turn Left & Success \\
3 & Turn Right & Success \\
4 & Stop & Success \\
\hline
\end{tabular}

Table 6. Detection distance of $25 \mathrm{~mm}$

\begin{tabular}{|c|c|c|}
\hline No & Type of command & $\begin{array}{l}\text { Detection } \\
\text { Result }\end{array}$ \\
\hline 1 & Forward & Fail \\
\hline 2 & Turn Left & Fail \\
\hline 3 & Turn Right & Fail \\
\hline 4 & Stop & Fail \\
\hline
\end{tabular}

Table 5. Detection distance of $23 \mathrm{~mm}$

\begin{tabular}{lll}
\hline No & Type of command & $\begin{array}{l}\text { Detection } \\
\text { Result }\end{array}$ \\
\hline 1 & Forward & Fail \\
2 & Turn Left & Fail \\
3 & Turn Right & Success \\
4 & Stop & Fail \\
\hline
\end{tabular}

Table 7. Detection distance of $30 \mathrm{~mm}$

\begin{tabular}{lll}
\hline No & Type of command & $\begin{array}{l}\text { Detection } \\
\text { Result }\end{array}$ \\
\hline 1 & Forward & Fail \\
2 & Turn Left & Fail \\
3 & Turn Right & Fail \\
4 & Stop & Fail \\
\hline
\end{tabular}

To validate the system, it is tested in actual working conditions. The system will be used to read RFId cards that have been arranged in such a way as to form the path that will be traversed by EDOT. The purpose of this test is to get the right delay in reading the RFId card and sending the command for EDOT to move according to the identity of the RFId card before reading the next card that has been arranged to form the path that EDOT will pass. The data shown in table 8 to table 12 below.

Table 8. Time Delay of 3 seconds

\begin{tabular}{lll}
\hline No & Order of the cards & Result \\
\hline 1 & F - F - F - S & Fail \\
2 & F - TR - F - S & Fail \\
3 & F - TR - TL - F - S & Fail \\
4 & F - TL - TR - S & Fail \\
\hline
\end{tabular}

Table 10. Time Delay of 0.75 second

\begin{tabular}{lll}
\hline No & Order of the cards & Result \\
\hline 1 & F - F - F - S & Fail \\
2 & F - TR - F - S & Fail \\
3 & F - TR - TL - F - S & Fail \\
4 & F - TL - TR - S & Fail \\
\hline
\end{tabular}

Table 12. Time Delay of 0.3 second

\begin{tabular}{lll}
\hline No & Order of the cards & Result \\
\hline 1 & F - F - F - S & Fail \\
2 & F - TR - F - S & Fail \\
3 & F - TR - TL - F - S & Fail \\
4 & F - TL - TR - S & Fail \\
\hline
\end{tabular}

Table 9. Time Delay of 1 second

\begin{tabular}{lll}
\hline No & Order of the cards & Result \\
\hline 1 & F - F - F - S & Fail \\
2 & F - TR - F - S & Fail \\
3 & F - TR - TL - F - S & Fail \\
4 & F - TL - TR - S & Fail \\
\hline
\end{tabular}

Table 11. Time Delay of 0.5 second

\begin{tabular}{lll}
\hline No & Order of the cards & Result \\
\hline 1 & F - F - F - S & Success \\
2 & F - TR - F - S & Success \\
3 & F - TL - F - S & Success \\
4 & F - TL - TR - F - S & Fail \\
\hline
\end{tabular}

\begin{tabular}{lcl}
\hline \multicolumn{2}{l}{ Abbreviation } & \\
\hline F & $:$ & Forward \\
TR & $:$ & Turn Right \\
TL & $:$ & Turn Left \\
S & $:$ & Stop \\
\hline
\end{tabular}




\section{International Journal of Applied Sciences and Smart Technologies}

Volume 1, Issue 2, pages 169-178

p-ISSN 2655-8564, e-ISSN 2685-9432

\section{Results and Discussion}

We shall present our research results and discussion in this section. The experiment data in table 2 to table 4 indicate that no error occur in path detecting process. Meanwhile, the experiment data in table 5 up to table 7 indicate that error occur in path detecting process. Since there is no problem with Arduino program, it is highly possible that the errors are mainly from the hardware configuration. The maximum detection distance is below $23 \mathrm{~mm}$. The performance of the path-tracking system can be improved by setting the RFId sensor distance to the RFId card in between $1 \mathrm{~mm}$ up to $20 \mathrm{~mm}$. The rigidity of the structure is also important to be taken into account for better result.

In the validation test, as we can see in the table 11 that the result of the test gives the best performance when the time delay was set to 0.5 second. Although in the fourth experiment it was not successful. There are several causes for this failure, such as the friction between the surface of the wheel and the trajectory, the thickness of the card that interfere the EDOT movement, etc.

From a number of experiments that have been carried out to produce conditions where the position of the RFId sensor must be installed at a distance of less than $23 \mathrm{~mm}$ from the surface of the track. Another parameter is setting the time delay. This will be used to adjust the time duration of the wheel to move after the sensor detects the track. The best delay time is around 0.5 seconds to get accurate results in reading RFId cards by RFId Sensors.

\section{Conclusion}

Design and development of a path-tracking system based on Radio Frequency Identification sensor for the Educational Toy Robot(EDOT) have been discussed in this paper. The path-tracking system has been tested by detecting the RFId cards in various distances and different command as well as by using real track out of arranged RFId cards. Based on data, some error still occurs in detecting processes. However, the source of the errors has been identified to be followed up. Improving the detecting quality of the path-tracking system may become the future research. 


\section{International Journal of Applied Sciences and Smart Technologies}

Volume 1, Issue 2, pages 169-178

p-ISSN 2655-8564, e-ISSN 2685-9432

\section{References}

[1] G. A. Demetriou, "Mobile robotics in education and research," in Mobile robots: Current trends, Z. Gacovski, Ed. Croatia: InTech, 27-48, 2011.

[2] D. A. N. Janis, D. Pang, and J. O. Wuwung, "Rancang bangun robot pengantar makanan line follower,” Jurnal Teknik Elektro dan Komputer, 3 (1), 1-10, 2014.

[3] Y. Prabowo and S. Hepy, "Line follower robot berbasiskan mikrokontroler ATMEL 16," Jurnal Ilmiah BIT, 8 (2) 44-52, 2011.

[4] F. A. Imbiri, N. Taryana, and D. Nataliana, "Implementasi sistem perparkiran otomatis dengan menentukan posisi parkir berbasis RFId," ELKOMIKA: Jurnal Teknik Energi Elektrik, Teknik Telekomunikasi, \& Teknik Elektronika, 4 (1), 31-46, 2016.

[5] N. Sparkhojayev and S. Guvercin, "Attendance control system based on RFIDtechnology," IJCSI International Journal of Computer Science Issues, 9 (3), 227-230, 2012. 
International Journal of Applied Sciences and Smart Technologies

Volume 1, Issue 2, pages 169-178

p-ISSN 2655-8564, e-ISSN 2685-9432

This page intentionally left blank 